\title{
Effects of hemodialysis on upper airways collapsibility in patients with chronic kidney disease
}

\author{
Israel Reis Santos', Aline Roberta Danaga², Anderson Soares Silva', Jessica Julioti Urbano', Ezequiel Fernandes Oliveira', \\ Roger Andre de Oliveira Peixoto', Letícia Lopes Guimarães', Patrícia Clemente Oliveira', Bruno Carlos de Queiroz², \\ Caroline de Freitas Gonçalves², Jéssica Scheurs Tomei², Eduardo Araújo Perez ${ }^{3}$, Sergio R Nacif4, Fatima Maria Leite de Souza ${ }^{5}$, \\ Luis Cuadrado Martin'ㄷㄴ Luis Vicente Franco Oliveira'
}

\begin{abstract}
Introduction: The Chronic Kidney Disease (CKD) is characterized as a clinical condition wherein the progressive loss of renal function for a period longer than months to years will lead to the onset of end-stage renal disease (ESRD). In the advanced stages of CKD, patients can only be treated with replacement therapy, dialysis or transplantation. In this context, hemodialysis (HD) presents as the main treatment option for these patients due to the lack of donors. Patients with ESRD in HD often present signs and symptoms related to pre-existing or acquired comorbidities with worsening of the disease. The most frequent are electrolytic disturbances, anemia, malnutrition, bone disease, Melittus diabetes, systemic arterial hypertension, gastrointestinal disorders, respiratory disorders, cardiovascular diseases, neurological changes and sleep disorders. Obstructive sleep apnea (OSA) presents a tenfold prevalence in patients with ESRD when compared to the general population, worsening the clinical symptoms and cardiovascular complications of this disease. The increase in the number of ESRD patients submitted to HD has assumed epidemic proportions worldwide. The causal association between sleep-disordered breathing, in particular OSA and ESRD, is still not well understood, which expands the possibility of new findings for the prevention, diagnosis and treatment of OSA, improving quality of life and reducing morbimortality. Objective: The present study involving ESRD patients undergone HD was designed with three main objectives: 1 ) to verify the prevalence and severity of sleep-disordered breathing; 2) to analyze the behavior of sleep apnea / hypopnea index (AHI) during the interdialitic period; 3) to verify weight gain and anthropometric measurements during the interdialitic period. Method: A consecutive single-center cross-sectional clinical study will be performed, double-blind, non-randomized to investigate the behavior of AHI in patients with ESRD undergoing HD in the nephrology unit (UNEFRO) at the Hospital of Santa Casa de Misericordia de Avaré, (SP), Brazil. The design and conduct of this study followed the guidelines of the Strengthening the Reporting of Observational Studies in Epidemiology (STROBE) statement.
\end{abstract}

Keywords: Sleep Disorders, Obstructive Sleep Apnea, Cardiorespiratory Monitoring, Quality of life.

Trial Registration: This protocol follows the STROBE (STrengthening the Reporting of OBservational studies in Epidemiology) and the study has been registered on ClinicalTrials.gov (NCT02390193).

\section{INTRODUCTION}

The Chronic Kidney Disease (CKD) is characterized as a clinical condition wherein the progressive loss of renal function for a period longer than months to years will lead to the onset of end-stage renal disease (ESRD). ${ }^{(1)}$

The CKD and ESRD have become a major public health problem worldwide, with a consequent increase in morbimortality and costs for the treatment of these diseases. In the United States, there are approximately 19 million adults with CKD and it is estimated that by the year 2030 more than two million people will require replacement therapy, dialysis or renal transplantation. ${ }^{(2)}$
In the advanced stages of CKD, patients can only be treated with replacement therapy, dialysis or transplantation. In this context, hemodialysis (HD) presents as the main treatment option for these patients due to the lack of donors. Approximately $65 \%$ of ESRD patients perform HD, $25 \%$ undergo renal transplantation and $10 \%$ are treated with peritoneal dialysis. ${ }^{(3)}$

In several countries of Europe, a high prevalence is also observed. In Germany, the prevalence of HD patients in 2009 was approximately 808 cases per million inhabitants. ${ }^{(4)}$ Epidemiological data from Brazil showed that in 2011 there

Corresponding author: Luis Vicente Franco Oliveira, PT, PhD. Laboratório do Sono, Universidade Nove de Julho (UNINOVE). Rua Vergueiro, 235/249, Liberdade, São Paulo, (SP), Brasil. Zip code 01504-001. E-mail: oliveira.lvf@uninove.br

${ }^{1}$ Rehabilitation Science Master's and PhD Degree Programs, Nove de Julho University (UNINOVE), São Paulo (SP) Brazil.

Full list of author information is available at the end of the article.

Financial support: Universidade Nove de Julho (Brazil), Foundation for Research Support of the State of São Paulo (local acronym FAPESP) and National Council of Scientific and Technological Development (local acronym CNPq). 
were more than 90.000 patients undergoing renal replacement therapy. ${ }^{(5)}$

Patients with ESRD in HD often present signs and symptoms related to pre-existing or acquired comorbidities with worsening of the disease. The most frequent are electrolytic disturbances, anemia, malnutrition, bone disease, diabetes Melittus (DM) ${ }^{(6)}$, systemic arterial hypertension $(\mathrm{SAH})^{(7,8)}$; gastrointestinal disorders; respiratory disorders ${ }^{(9)}$; cardiovascular diseases ${ }^{(10-12)}$; neurological changes ${ }^{(13)}$; and sleep disorders. ${ }^{(14)}$

The prevalence of sleep disturbances in ESRD in HD is considerable. Approximately $50-80 \%$ of these patients have sleep complaints, including insomnia, sleep apnea (central and / or obstructive), restless legs syndrome and excessive daytime sleepiness (ESD)..$^{(15-18)}$

The obstructive sleep apnea (OSA) presents a tenfold prevalence in patients with ESRD when compared to the general population, worsening the clinical symptoms and cardiovascular complications of this disease.${ }^{19}$ OSA is a serious clinical pathological condition characterized by repeated episodes of absence of airflow caused by total collapse of the upper airway for at least 10 seconds in the presence of ventilatory effort during sleep, usually accompanied by snoring, nocturnal hypoxemia and EDS.(20)

Some studies have shown that OSA is more common in pathologies characterized by edemas, such as heart failure and renal failure. ${ }^{(21-23)}$ One of the causes is due to the redistribution of intravascular and interstitial fluid from the lower limbs to the thoracic and cervical region, increasing the extraluminal pressure in the upper airway when patients assume the position of decubitus, especially when they go to sleep. Therefore, it has been shown that the displacement of rostral fluid during sleep contributes to the pathogenesis of OSA. (24-27)

The increase in the number of ESRD patients submitted to HD has assumed epidemic proportions worldwide. The negative impact on the quality of life and morbimortality caused by cardiorespiratory dysfunctions and sleep disorders is already well established in the literature. The causal association between sleep-disordered breathing, in particular OSA and ESRD, is still not well understood, which expands the possibility of new findings for the prevention, diagnosis and treatment of OSA, improving quality of life and reducing morbimortality.

\section{Aims and Hypotheses}

The present study involving ESRD patients undergone HD was designed with three main objectives: 1) to verify the prevalence and severity of sleep-disordered breathing; 2) to analyze the behavior of sleep apnea / hypopnea index $(\mathrm{AHI})$ during the interdialitic period; 3 ) to verify weight gain and anthropometric measurements during the interdialitic period. According to the existing literature, we can hypothesize that $\mathrm{AHI}$ will vary during the interdialytic period in ESRD patients. ${ }^{(24-27)}$

\section{METHODS}

\section{Study design and setting}

A consecutive single-center cross-sectional clinical study will be performed, double-blind, non-randomized to investigate the behavior of $A H I$ in patients with ESRD undergoing HD in the nephrology unit (UNEFRO) at the Hospital of Santa Casa de Misericordia de Avaré, (SP), Brazil. The design and conduct of this study followed the guidelines of the Strengthening the Reporting of Observational Studies in Epidemiology (STROBE) statement, according to figure 1.

\section{Participants}

The subjects, patients diagnosed with ESRD undergoing regular HD, between 07:00 and 17:00h, 3 times a week, will be invited to participate in the study, consecutively and

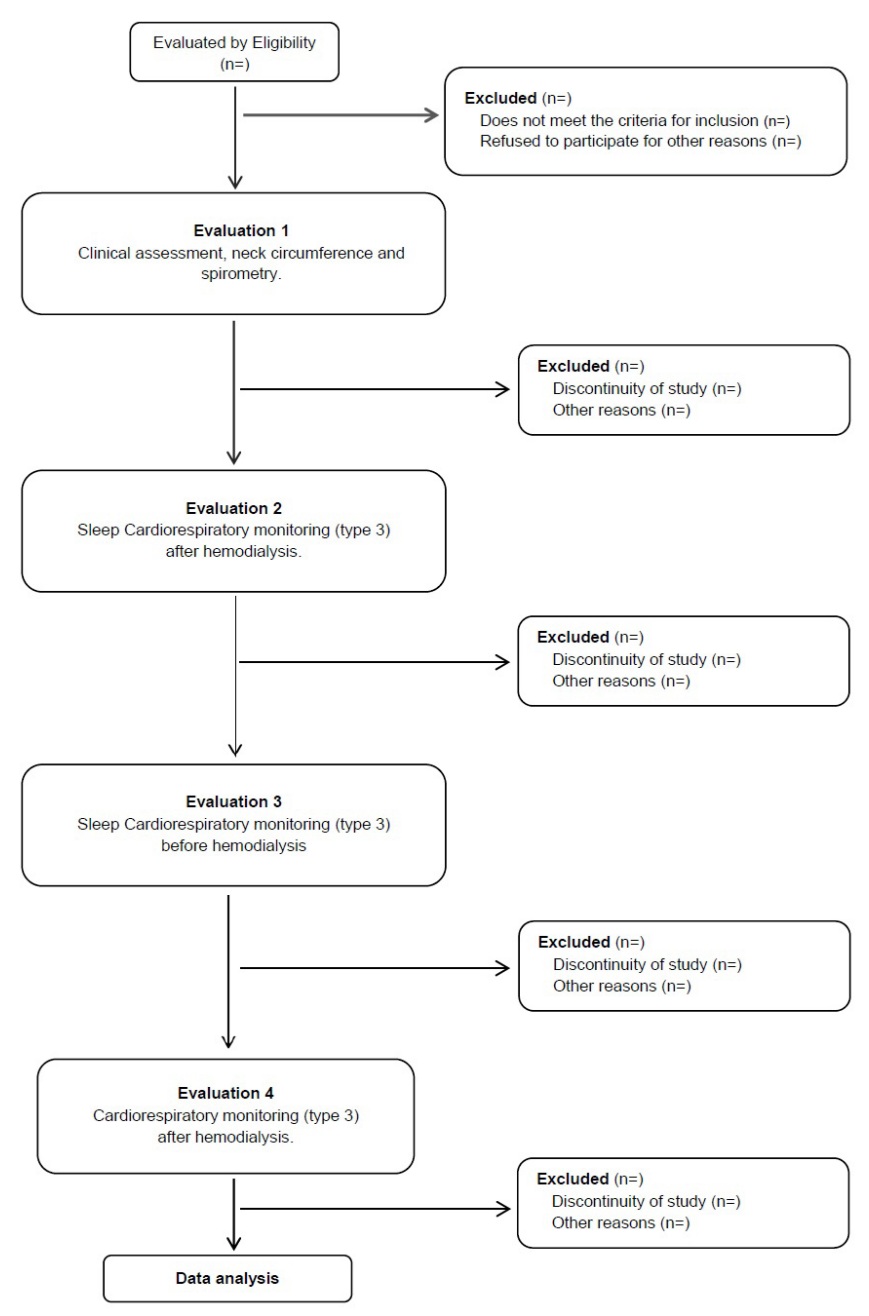

Figure 1. Flowchart of the study. 
selected for eligibility in accordance with the proposed criteria. Inclusion criteria are patients of both sexes, aged between 18 and 70 years, candidates for renal transplantation, with sufficient cognitive level to understand the procedures and follow the instructions of the free and informed consent term. Will be excluded patients with craniofacial abnormalities, undergoing active treatment of OSA, had an left ventricle ejection fraction of $\leq 45 \%$ on routine echocardiography, active malignant disease, abusive use of alcohol and / or illicit drugs, dementia or treatment of psychiatric illnesses leading to an inability to provide informed consent.

\section{Variables}

Primary outcome

Variation of $\mathrm{AHI}$ during the interdialytic period in ESRD patients.

\section{Secondary outcome}

Prevalence and severity of sleep-disordered breathing in ESRD patients submitted to HD.

\section{Ethical considerations}

The study complied with the Declaration of Helsinki, Guidelines and Rules for Research Involving Human Beings of the Brazil National Health Council of the Ministry of Health (December, 2012). This protocol received approval from the Human Research Ethics Committee of Universidade Nove de Julho (Brazil) under process no. 368856/2010 and was registered with ClinicalTrials.gov identifier NCT02390193. All participants provided written informed consent.

\section{Renal Replacement Therapy}

\section{Hemodialysis}

Regular daytime HD will be standardised during the trial. It was performed 3 times per week, with a $4 \mathrm{~h}$ session duration, $250 \mathrm{~mL} / \mathrm{min}$ blood flow, and $500 \mathrm{~mL} / \mathrm{min}$ dialysate flow, using bicarbonate buffered dialysate with $1.25 \mathrm{mmol} / \mathrm{L}$ ionised calcium concentration, dialysate temperature of $36.5^{\circ} \mathrm{C}$, and Polyflux $17 \mathrm{~L}$ dialyser. The ultrafiltration amount for each haemodialysis session was decided by individual dry weight, which was fixed during the trial. In addition, during the trial, the patients were not permitted to change their medication or start new drugs, especially antiplatelet agents, angiotensin-converting enzyme inhibitors, angiotensin II receptor antagonists, calcium channel blockers, and $\beta$ blockers. The patients who required a change in medication for medical reasons were subsequently excluded from the study. Use of $\mathrm{N}$ acetylcysteine was also prohibited in view of its potential influence on ischemia reperfusion injury. ${ }^{(28)}$

\section{Study Protocol}

Initially, patients underwent a medical consultation with anamnestic deepening to identify the complaints of sleep disorders and anthropometric evaluation. The clinical variables, such as comorbidities, dialysis time and renal disease etiology, and biochemical analysis of blood will be obtained from patients records. All subjects involved in this study should be clinically stable for at least three months.

\section{Clinical Evaluation}

The clinical evaluation will be performed by a doctor and a physiotherapist, including clinical history, check waist circumference and neck. For the calculation of body mass index (BMI) by weight / height ${ }^{2}$. The body weight $(\mathrm{kg})$ and height $(\mathrm{cm})$ will be verified through an electronic scale (model 200/5, Welmy Indústria e Comércio Ltda, São Paulo, Brazil)(29) Cardiac and respiratory rates and peripheral blood pressure will also be checked.

\section{Waist and neck circumferences}

The measurement of the circumferences will be performed with the patients in orthostatic position, using a non-elastic tape measure, with precision of $0.1 \mathrm{~cm}$ and $7 \mathrm{~mm}$ of width, parallel to the ground. The anatomical points for the measurements will be standardized. The waist circumference will be measured at the midpoint between the lower edge of the last rib and the iliac crest and the circumference of the neck will be checked horizontally on the cricoid cartilage. ${ }^{(30)}$

\section{Sleep Evaluation}

\section{Nocturnal Polygraphy}

Nocturnal Home Polygraphy (PG) will be performed on the night after HD and on two subsequent nights using an type 3 portable monitoring Apnea Link Air device (ResMed Corporation, San Diego, CA, USA) including a five record signals of respiratory effort, respiratory flow, snore, blood oxygen saturation and pulse. ${ }^{(31)}$ All respiratory events were manually scored by the same experienced pulmonologist, specialist in Sleep Medicine, according to the American Academy of Sleep Medicine criteria. The AHI will be calculated as the number of apneas and hypopneas per hour of recording, and OSA diagnosis was retained for $\mathrm{AHI} \geq 5 / \mathrm{h}^{(32)}$

\section{Quality control}

To ensure data quality, all health professionals involved in this study will receive specific training. A periodic external monitoring will be performed to verify the adequate development of the proposed protocol.

\section{Sample Calculation}

Sample size was calculated according to a previously published clinical study ${ }^{(33)}$ where the physiological sleep variables were analyzed in 20 patients with ESRD before and after HD 
through PSG, with a prevalence of $85 \%$ of OSA being observed using a cutt off $>15 \mathrm{AHI}$. The sample power test was calculated in 19 ESRD patients, adopting a 95\% level of significance, with an error $\alpha$ of $5 \%$ and a error $\beta=90 \%$ (power test).

\section{Statistical analysis}

Statistical analysis will be conducted using SPSS version 22 (IBM, Chicago, IL, EUA). The Shapiro-Wilk test will be performed to verify the normality of the data. Mean and SD will be used to describe continuous variables, and percentages were used to describe categorical variables. Will be used a paired t test or McNemar test to compare the measurements performed before and after hemodialysis. Associations between continuous variables will be assessed by linear regression. Will be conducted univariate linear regressions to explore the factors associated with the severity of OSA. To further investigate the association between OSA and fluid overload, we will also compare the characteristics of participants in whom hemodialysis effectively reduced fluid overload in a post hoc analysis or chi-squared and $t$ tests. Statistical significance will be establish at $p$ value 0.05 .

\section{Description of risks}

There will be no risks for included patients.

\section{Acknowledgements}

The Sleep Lab receives funding from the Universidade Nove de Julho (Brazil) and research projects approved by the Brazilian agencies promoting the Foundation for Research Support of the State of São Paulo (local acronym FAPESP; Protocol no 2003 / 01810-4) and LVFO receives the productivity in research modality IB subsidies by National Council of Scientific and Technological Development (local acronym CNPq; process no 313.053 / 2014-6). EFO receives subsidies from the Foundation for Research Support of the State of São Paulo (local acronym FAPESP: Protocol no 2015 / 11738-6)

\section{AUTHOR CONTRIBUTIONS}

LVFO and IRS defined the concept of the study, created the hypotheses and wrote the original proposal of the study protocol. EFO, ASS, JJU, ARD, BCQ and LVFO contributed significantly to the writing of this proposal. JJU, EAP, IRS, RAOP, SRN, LLG, ASS, e FMLS, LCM, CFG, JST, PCO, IRS and LVFO were involved in the critical review of the manuscript and final writing. All authors read and approved the final manuscript.

\section{CONFLICTS OF INTEREST}

The are no conflicts of interest.

\section{AUTHOR DETAILS}

${ }^{2}$ Department of Physiotherapy, Faculdade Sudoeste Paulista (FSP), Avare, SP, Brazil.

${ }^{3}$ Research in Surgery Master's and PhD Degree Programs, Faculdade de Ciências Médicas da Santa Casa de São Paulo (SP), Brazil.

${ }^{4}$ Hospital Servidor Público Estadual (HSPE), São Paulo (SP), Brazil.

${ }^{5}$ Nephrology Unit (UNEFRO), Santa Casa de Misericordia de Avare, Avare, SP, Brazil.

${ }^{6}$ Department of Internal Medicine of Botucatu Medical School, Sao Paulo State
University (UNESP), Botucatu, SP, Brazil.

\section{REFERENCES}

1- Kalamas AG, Niemann CU Patients with Chronic Kidney Disease. Med Clin N Am 2013;97:1109-1122.

2- Coresh J, Byrd-Holt D, Astor BC, Briggs JP, Eggers PW, Lacher DA, et al. Chronic Kidney Disease Awareness, Prevalence, and Trends among U.S. Adults, 1999 to 2000. J Am Soc Nephrol 2005;16:180 -188.

3- Zoccali C, Kramer A, Jager KJ. Epidemiology of CKD in Europe: an uncertain scenario. Nephrol Dial Transplant 2010;25:1731-1733.

4- Abbasi M, Chertow GM, Hall YN. End-stage renal disease. BMJ Clinical Evidence 2010;07:2002.

5- Sesso, RC, Lopes AA, Thomé FS, Lugon Jr, Watanabe Y, Santos Dr. Chronic dialysis in Brazil: report of the Brazilian dialysis census, 2011. J Bras Nefrol 2012;34(3):272-7.

6- Jurkovitz CT, Elliott D, Li S, Saab G, Bomback AS, Norris KC, et al. Physician utilization, risk-factor control, and CKD progression among participants in the Kidney Early Evaluation Program (KEEP). Am J Kidney Dis 2012;59(2):S24-33.

7- Nieto FJ, Young TB, Lind BK, Shahar E, Samet JM, Redline S, et al. Association of sleep-disordered breathing, sleep apnea and hypertension in a large community-based study. Sleep Heart Health Study. JAMA 2000;12(14):1829-1836.

8- Drager LF, Bortolotto LA, Krieger EM, Lorenzi-Filho G. Additive Effects of Obstructive Sleep Apnea and Hypertension on Early Markers of Carotid Atherosclerosis. Hypertension 2009;53(1):64-69.

9- Mclntyre CW, Selby NM, Sigrist M, Pearce LE, Mercer TH, Naish PF. Patients receiving maintenance dialysis have more severe functionally significant skeletal muscle wasting than patients with dialysis-independent chronic kidney disease. Nephrol Dial Transplant 2006;21(8):2210-2216.

10- London GM. Cardiovascular Disease in Chronic Renal Failure: Pathophysiologic Aspects. Semin Dial 2003;16(2):85-94.

11- Weiner DE, Tighiouart H, Stark PC, Amin MG, Macleod B, Griffith JL, et al. Kidney Disease as a Risk Factor for Recurrent Cardiovascular Disease and Mortality. Am J Kidney Dis. v.44, n.2, p.198-206, 2004.

12- Zoccali C, Mallamaci F, Tripepi G. Novel Cardiovascular Risk Factors in End-Stage Renal Disease. J Am Soc Nephrol 2004;15(Suppl 1):S77-S80.

13- Brouns R, De Deyn P. Neurological complications in renal failure: a review. Clin Neurol Neurosurg. 2004;107(1):1-16.

14- Turek NF, Ricardo AC, Lash JP. Sleep disturbances as nontraditional risk factors for development and progression of CKD: review of the evidence. Am J Kidney Dis. 2012;60(5):823-33.

15- Hamdan Al-Jahdali. Prevalence of Sleep Apnea and Excessive Day Time Sleepiness in Patients with End-Stage Renal Disease on Dialysis. Saudi J Kidney Dis Transpl 2012;23(2):251-261.

16- Merlino G, Piani A, Dolso P, Adorati M, Cancelli I, Valente M, Gigli GL. Sleep disorders in patients with end-stage renal disease undergoing dialysis therapy. Nephrol Dial Transplant 2006;21:184-190.

17- Unruh ML, Sanders MH, Redline S. Sleep Apnea in Patients on Conventional Thrice-Weekly Hemodialysis: Comparison with Matched Controls from the Sleep Heart Health Study. J Am Soc Nephrol 2006;17:3503-3509.

18- Ogna VF, Ogna A, Pruijm M, Bassi I, Zuercher E, Halabi G, et al. Prevalence and Diagnostic Approach to Sleep Apnea in Hemodialysis Patients: A Population Study. Biomed Res Int. 2015;2015:103686.

19- Wali SO, Alkhouli A, Howladar M, Ahmad I, Alshohaib S, Al-Ghamdi S, et al. Risk of obstructive sleep apnea among Saudis with chronic renal failure on hemodialysis. Ann Thorac Med. 2015 Oct-Dec;10(4):263-8.

20- Sleep-related breathing disorders in adults: Recommendations for syndrome definition and measurement techniques in clinical research. The Report of an American Academy of Sleep Medicine Task Force. SLEEP, 1999, 22, 5,667-89. 
21- Javaheri S, Parker TJ, Wexler L, Michaels SE, Stanberry E, Nishyama H, et al. Occult sleep-disordered breathing in stable congestive heart failure. Ann Intern Med. 1995;122(7):487-492.

22- Javaheri S, Parker TJ, Liming JD, Corbett WS, Nishiyama H, Wexler $\mathrm{L}$, et al. Sleep apnea in 81 ambulatory male patients with stable heart failure. Types and their prevalences, consequences, and presentations. Circulation. 1998 ;97(21):2154-2159.

23- Shahar E, Whitney CW, Redline S, Lee ET, Newman AB, Nieto FJ, et al. Sleep-disordered breathing and cardiovascular disease: cross-sectional results of the Sleep Heart Health Study. Am J Respir Crit Care Med. 2001;163(1):19-25.

24- Redolfi S, Yumino D, Ruttanaumpawan P, Yau B, Su MC, Lam J, et al. Relationship between overnight rostral fl uid shift and Obstructive Sleep Apnea in nonobese men. Am J Respir Crit Care Med. 2009;179(3):241246.

25- Elias RM, Bradley TD, Kasai T, Motwani SS, Chan CT et al. Rostral overnight fluid shift in end-stage renal disease: relationship with obstructive sleep apnea. Nephrol Dial Transplant 2011; 27: 1569-1573.

26- Elias RM, Chan CT, Paul N, Motwani SS, Kasai T, Gabriel JM, et al. Relationship of pharyngeal water content and jugular volume with severity of obstructive sleep apnea in renal failure. Nephrol Dial Transplant (2013) 28: 937-944.
27- Lyons OD, Chan CT, Yadollahi A, Bradley TD. Effect of Ultrafiltration on Sleep Apnea and Sleep Structure in Patients with End-Stage Renal Disease. Am J Respir Crit Care Med 2015;191(11):1287-1294.

28- Park J, Ann SH, Chung HC, Lee JS, Kim SJ, Garg S, et al. Remote ischemic preconditioning in hemodialysis: a pilot study. Heart Vessels. 2014 Jan;29(1):58-64.

29- World Health Organization. Obesity: preventing and managing the global epidemic. Report of a World Health Organization Consultation. Geneva: World Health Organization, 2000. p. 256. WHO Obesity Technical Report Series, n. 284.

30- Gabrielsen AM, Lund MB, Kongerud J, Viken KE, Roislien J, Hjelmesaeth J. The relationship between anthropometric measures, blood gases, and lung function in morbidly obese white subjects. Obes Surg 2011;21(4):485-491.

31- Collop NA, Anderson WM, Boehlecke B, Claman D, Goldberg R, Gottlieb DJ, et al. Clinical guidelines for the use of unattended portable monitors in the diagnosis of obstructive sleep apnea in adult patients. J Clin Sleep Med 2007;3(7):737-747.

32- Berry RB, Brooks R, Gamaldo CE, Harding SM, Lloyd RM, Marcus CL, et al. The AASM Manual for the Scoring of Sleep and Associated Events: Rules, Terminology and Technical Specifications, Version 2.2. www.aasmnet.org. Darien, Illinois: American Academy of Sleep Medicine, 2015.

33- Ogna A, Forni Ogna V, Mihalache A, Pruijm M, Halabi G, Phan O, et al., Obstructive Sleep Apnea Severity and Overnight Body Fluid Shift before and after Hemodialysis. Clin J Am Soc Nephrol 10: 1002-1010, 2015. 